\title{
Adaptive Energy Efficiency Maximization for Cognitive Underwater Acoustic Network under Spectrum Sensing Errors and CSI Uncertainties
}

\author{
Yaohui $W u\left(\mathbb{D},{ }^{1,2}\right.$ Youming $L i \mathbb{D}^{2},{ }^{2}$ and Qingpeng Yao ${ }^{1}$ \\ ${ }^{1}$ School of Electronic and Information Engineering, Zhejiang Wanli University, Ningbo 315100, China \\ ${ }^{2}$ College of Information Science and Engineering, Ningbo University, Ningbo 315211, China
}

Correspondence should be addressed to Youming Li; liyouming@nbu.edu.cn

Received 14 December 2018; Revised 22 April 2019; Accepted 12 May 2019; Published 23 May 2019

Academic Editor: Donatella Darsena

Copyright (C) 2019 Yaohui Wu et al. This is an open access article distributed under the Creative Commons Attribution License, which permits unrestricted use, distribution, and reproduction in any medium, provided the original work is properly cited.

\begin{abstract}
Energy efficiency (EE) maximization problem for Cognitive Underwater Acoustic Network is investigated in this study. Available works on EE usually assume that spectrum sensing is accurate or that channel state information (CSI) is perfect, which is often impractical. Thus, an adaptive resource allocation scheme is proposed to maximize the EE, subject to the transmission power constraint of secondary user (SU) and the interference power constraint of primary user (PU). By taking the spectrum sensing errors into account, we add power interference from PU to SU in the objective function. Besides, interference tolerance factor is introduced to control the interference from SU to PU. Assuming CSI uncertainties of the involved channels are bounded, they are separately modeled as stochastic-case or worst-case according to their nature. Since the established optimization problem is nonconvex, it is converted into a convex one and then solved by the techniques of fractional programming and dual decomposition. Simulation results validate that the EE can be improved by classifying the CSI uncertainties and solving the expectation of the CSI correlation function. Furthermore, the interference from SU to PU can be controlled well by the adjustment of the interference tolerance factor.
\end{abstract}

\section{Introduction}

In recent years, underwater acoustic network (UAN) has become widely accepted in areas such as oceanographic environmental monitoring, offshore exploration, and assisted navigation [1]. However, the underwater acoustic environment is usually faced with high spectrum competition among different users including UAN users, sonar users, and marine mammals. To improve the efficiency of spectrum utilization, cognitive underwater acoustic network (CUAN) is proposed to achieve the environment-friendly transmission [2]. Generally, there exist three access strategies in CUAN: underlay, interweave, and overlay [3]. In underlay strategy, secondary user (SU) can always share the same channel with primary user (PU) conditioned that the SU interference to PU is below the threshold, and the condition has recently been relaxed by convolutive superposition method [4]. In the interweave approach, SU opportunistically transmits the data with two power levels according to the channel state (e.g., busy or idle).
In overlay strategy, SU should sense the channel in advance and then occupy the idle channel to transmit the data. In this study, the overlay strategy in CUAN is adopted owing to its efficient cooperation between SU and PU [5].

Energy efficiency (EE) is an important objective in CUAN since most underwater devices are constrained by energy supply and difficult to resupply [6]. Thus, resource allocation design for EE maximization, which not only satisfies the limited transmission power but also considers the operational expenditure, is needed urgently for CUAN. In [7], a power allocation method named water-filling factors aided search is proposed to optimize the system EE. To avoid the spectrum competition between SU and PU, priority based multiresource allocation scheme is proposed to maximize each SU's EE in [8]. To decrease the energy consumption, an optimization process is presented in [9], which considers the code rate in conjunction with the selection of the modulation order. To improve the EE of CUAN using an expanded bandwidth, carrier aggregation scheme is proposed in [10], 
where multiple carriers are aggregated for one transmitter to transmit data.

The aforementioned works assume that spectrum sensing is accurate and that perfect channel state information (CSI) is available at the transmitter of SU. However, it is unreasonable due to the imprecision and randomness of CUAN channels. To deal with the CSI uncertainties, adaptive optimization has been applied recently. Using the statistical knowledge of the channel uncertainties, a stochastic scheme is formulated under the interference power constraint in [11]. To strictly satisfy the predefined threshold of PU even in the presence of channel errors, worst-case optimization approach is proposed in [12]. On the other hand, spectrum sensing errors have been paid more attention to in the resource allocation. In [13], computationally efficient suboptimal algorithms are proposed under the interference that arises as a result of imperfect spectrum sensing. To handle the spectrum sensing errors and the mutual interference between SU and PU, a suboptimal power and subcarrier allocation algorithm is proposed in [14].

In fact, there are few works simultaneously involved in spectrum sensing errors and CSI uncertainties. Although an adaptive rate maximization method under spectrum sensing errors and channel uncertainties is introduced in [15], it simply handles all the involved channel uncertainties by the worst-case; besides, its optimization object is capacity rather than EE.

In this paper, an adaptive EE maximization scheme for CUAN is proposed by dealing with both spectrum sensing errors and CSI uncertainties. To comprehensively investigate the impact of CSI uncertainties on system performance, we separately model the uncertainties of the involved channels as stochastic-case or worst-case according to their nature. By taking the spectrum sensing errors into account, we add power interference from PU to SU in the objective function. Besides, interference tolerance factor is introduced into the constraint to control the interference from SU to PU. The main contributions can be summarized as follows:

(i) CSI uncertainties are considered and modeled in all the involved channels: (1) the channel gain from SU-transmitter (SU-Tx) to SU-receiver (SU-Rx); (2) the channel gain from SU-Tx to PU-Rx; (3) the channel gain from PU-Tx to SU-Rx. The last two types are modeled as worst-case to improve the system robustness, while the first is modeled as stochasticcase to improve the system EE.

(ii) Spectrum sensing errors are considered in the EE maximization problem. Meanwhile, by introducing interference tolerance factor into the constraint, we can control the interference from SU to PU well.

(iii) Owing to the fact that the EE maximization problem is nonconvex, it is converted into a convex form by fractional programming technique and then solved by dual decomposition method.

The rest of this paper is structured as follows. The system model in overlay CUAN is discussed in Section 2, and an adaptive EE maximization problem is formulated in

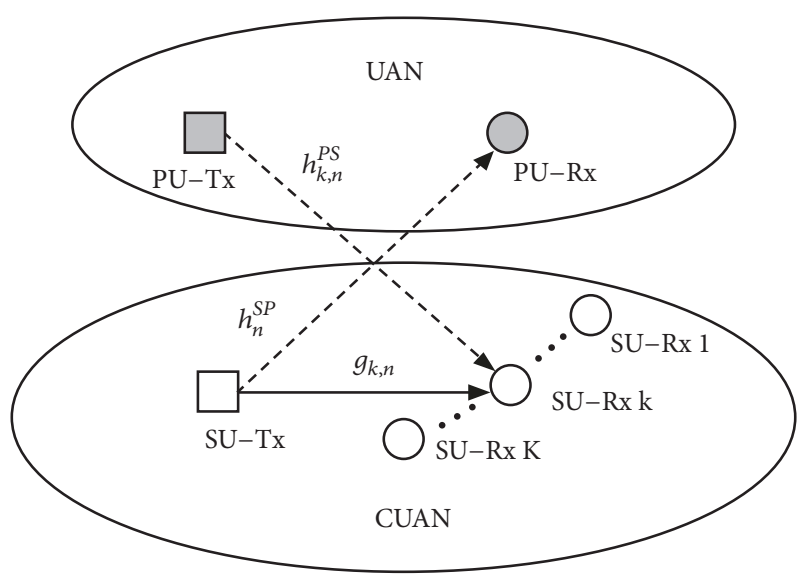

Figure 1: A downlink overlay CUAN model.

Section 3. Then, the optimization problem is transformed and solved in Section 4, followed by the proposed algorithm in Section 5. The numerical results and analysis are illustrated in Section 6. Finally, the conclusion is drawn in Section 7.

\section{Channel and System Models}

In this paper, the overlay strategy is adopted owing to its efficient cooperation between SU and PU. A downlink overlay CUAN is considered as shown in Figure 1, where there are $K$ pairs of SU Tx/Rx coexisting with one pair of $\mathrm{PU} T \mathrm{Tx} / \mathrm{Rx}$. The CUAN is assumed to employ orthogonal frequency division multiplexing access (OFDMA) scheme. To withstand the multipath fading, the spectrum band $B$ is divided into $J$ subcarriers to satisfy that the subcarrier bandwidth $B / J$ is less than the channel coherence bandwidth; then each subcarrier will experience flat fading in each OFDMA symbol period [16]. SU can opportunistically occupy the idle subcarriers by spectrum sensing. Let $N$ denote the number of subcarriers occupied by SU, where $N \leq J$. Let $g_{k, n}$ denote the direct channel gain from SU-Tx to the $k$ th SU-Rx in the $n$th subcarrier. The channel gain from SU-Tx to PU-Rx in the $n$th subcarrier is denoted by $h_{n}^{S P}$, and $h_{k, n}^{P S}$ is the channel gain from PU-Tx to the $k$ th SU-Rx in the $n$th subcarrier.

2.1. Channel Model. In CUAN, the channel gain $g_{k, n}$ has its frequency response as $[17,18]$

$$
g_{k, n}=\frac{1}{\sqrt{Q\left(d_{k}, f_{n}\right)} \sum_{l=1}^{L} a_{l} e^{-j 2 \pi\left(f_{n}+\Delta f_{l}\right) \tau_{l}}}
$$

where $d_{k}$ is the transmission distance from SU-Tx to the $k$ th SU-Rx, $f_{n}$ is the center frequency of the $n$th subcarrier, and $Q\left(d_{k}, f_{n}\right)$ is the corresponding attenuation. $L$ is the number of multiple paths, $\Delta f_{l}$ is the Doppler shift of the $l$ th path, and $a_{l}$ and $\tau_{l}$ are the amplitude and delay of the corresponding channel response, respectively.

In formula (1), the attenuation $Q\left(d_{k}, f_{n}\right)$ can be given as

$$
Q\left(d_{k}, f_{n}\right)=A_{0} d^{s} a\left(f_{n}\right)^{d_{k}}
$$


where $A_{0}$ is a unit-normalizing constant and $s$ is the spreading factor. $a\left(f_{n}\right)$ is the absorption coefficient which can be expressed empirically by the Thorps formula in $\mathrm{dB} / \mathrm{km}$ for $\mathrm{f}$ in $\mathrm{kHz}$ as

$$
\begin{aligned}
10 \log _{10} a\left(f_{n}\right)= & 0.11 \frac{f_{n}^{2}}{1+f_{n}^{2}}+\frac{44 f_{n}^{2}}{4100+f_{n}^{2}}+2.75 \\
& \times 10^{-4} f^{2}+0.003
\end{aligned}
$$

If only the corresponding transmission distance is substituted in (1), the channel gain $h_{k, n}^{P S}$ and $h_{n}^{S P}$ can be similarly calculated.

In CUAN, the ambient noise can be modeled using four sources: waves, turbulence, shipping, and thermal noise. In the range of interest, the overall power spectral density (p.s.d.) of the noise in $\mathrm{dB}$ in the frequency $f_{n}$ can be approximated as [19]

$$
10 \log _{10} N_{t}\left(f_{n}\right)=\chi_{0}-18 \log _{10} f_{n}
$$

where $f_{n}$ is in $\mathrm{kHz}$, and $\chi_{0}$ is the noise constant level related to the specific deployment site. It can be seen that the overall p.s.d. of the noise decays with frequency.

2.2. System Model. Due to the spectrum sensing errors, the subcarrier used by SU may also be occupied by PU. Let $O_{n}$ and $I_{n}$ denote the state that the $n$th subcarrier is actually occupied by PU or not, respectively. Let $\widehat{O}_{n}$ and $\widehat{I}_{n}$ denote the spectrum sensing value of $O_{n}$ and $I_{n}$, respectively. Let $\beta_{n}$ denote the probability that SU identifies the $n$th subcarrier as idle but it is actually occupied by PU (i.e., spectrum sensing error occurring in the $n$th subcarrier). Then $\beta_{n}$ can be given as

$$
\begin{aligned}
\beta_{n} & =P\left(O_{n} \mid \widehat{I}_{n}\right)=\frac{P\left(O_{n}, \widehat{I}_{n}\right)}{P\left(\widehat{I}_{n}\right)} \\
& =\frac{P\left(\widehat{I}_{n} \mid O_{n}\right) P\left(O_{n}\right)}{P\left(O_{n}\right)\left(P\left(\widehat{I}_{n} \mid O_{n}\right)\right)+\left(1-P\left(O_{n}\right)\right)\left(P\left(\widehat{I}_{n} \mid I_{n}\right)\right.} \\
& =\frac{P\left(O_{n}\right)\left(1-P_{d}\right)}{P\left(O_{n}\right)\left(1-P_{d}\right)+\left(1-P\left(O_{n}\right)\right)\left(1-P_{f a}\right)}
\end{aligned}
$$

where $P(\cdot)$ is the probability operator, and $P_{d}$ and $P_{f a}$ are the detection probability and false-alarm probability, respectively.

On the other hand, due to the CSI uncertainties, the channel gains need to be modified as follows:

$$
\begin{gathered}
\widehat{g}_{k, n}=g_{k, n}\left(1+e_{k, n}\right) \\
\widehat{h}_{k, n}^{P S}=h_{k, n}^{P S}\left(1+e_{k, n}^{P S}\right) \\
\widehat{h}_{n}^{S P}=h_{n}^{S P}\left(1+e_{n}^{S P}\right)
\end{gathered}
$$

where $\widehat{g}_{k, n}, \widehat{h}_{k, n}^{P S}$, and $\widehat{h}_{n}^{S P}$ are the actual values of the channel gains, respectively. Correspondingly, $e_{k, n}, e_{k, n}^{P S}$, and $e_{n}^{S P}$ are the CSI uncertainties.
Assuming the uncertainties are bounded [20], their regions can be modelled as follows:

$$
\begin{aligned}
& \left|e_{k, n}\right| \leq \theta_{k, n} \\
& \left|e_{k, n}^{P S}\right| \leq \omega_{k, n} \\
& \left|e_{n}^{S P}\right| \leq \varphi_{n}
\end{aligned}
$$

where $\theta_{k, n}, \omega_{k, n}$, and $\varphi_{n}$ denote the uncertainty size of the involved channels (USIC), respectively.

As a consequence of the spectrum sensing errors and CSI uncertainties, the rate of the $k$ th $\mathrm{SU}$ in the $n$th subcarrier should be expressed as

$$
\begin{aligned}
R_{k, n}= & \left(1-\beta_{n}\right) E\left\{\log _{2}\left(1+\frac{p_{k, n}\left|\widehat{g}_{k, n}\right|^{2}}{\sigma_{n}^{2} \Gamma}\right\}\right. \\
& +\beta_{n} E\left\{\log _{2}\left(1+\frac{p_{k, n}\left|\widehat{g}_{k, n}\right|^{2}}{\left(q_{k, n}^{P S}\left|\widehat{h}_{k, n}^{P S}\right|^{2}+\sigma_{n}^{2}\right) \Gamma}\right\}\right.
\end{aligned}
$$

where $E\{\cdot\}$ is the expectation operator, $\sigma_{n}^{2}=N_{t}\left(f_{n}\right) B / J$ is the overall noise in the $n$th subcarrier, $p_{k, n}$ is the allocated power from SU-Tx to the kth SU-Rx in the nth subcarrier, and $q_{k, n}^{P S}$ is the interference power coming from PU-Tx. $\Gamma=-\ln (5 B E R) / 1.5$ is the signal-to-noise ratio (SNR) gap between the theoretical channel capacity and the multilevel quadrature amplitude modulation scheme, which depends on the target bit error rate (BER). $p_{k, n}\left|\widehat{g}_{k, n}\right|^{2} /\left(q_{k, n}^{P S}\left|\widehat{h}_{k, n}^{P S}\right|^{2}+\sigma_{n}^{2}\right) \Gamma$ and $p_{k, n}\left|\widehat{g}_{k, n}\right|^{2} / \sigma_{n}^{2} \Gamma$ represent the SNR under the spectrum sensing error or not, respectively.

Note that formula (12) is based on OFDMA system, and the spectrum band is divided into a number of subcarriers. Let the subcarrier bandwidth be less than the channel coherence bandwidth; then each subcarrier experiences flat fading; i.e., the actual values of the channel gains $\widehat{g}_{k, n}$ and $\widehat{h}_{k, n}^{P S}$ remain unchanged in each OFDMA symbol period. Due to the multipath fading of the underwater acoustic channel as seen in formula (1), the channel gain is different for different user and different subcarrier; hence there is one user $k^{*}$ for subcarrier $n$, and the value of $\widehat{g}_{k^{*}, n}$ is the highest. Assuming that other conditions are the same, the optimal rate of subcarrier $n$ can be achieved by assigning subcarrier $n$ to user $k^{*}$. However, the system model consisting of $R_{k, n}$ is more complex, owing to the CSI uncertainties of $\widehat{g}_{k, n}$ and $\widehat{h}_{k, n}^{P S}$, the randomness of $\beta_{n}$ caused by the spectrum sensing errors, and the power consumption consideration of $p_{k, n}$.

\section{Problem Formulation}

Due to the spectrum sensing errors or CSI uncertainties, adaptive problems have been presented in many researches, but few works have considered the two aspects together. In this section, a more overall adaptive EE maximization problem will be formulated. 
To maximize the EE of CUAN while guaranteeing the PU's interference threshold, the resource allocation problem can be formulated as follows:

$$
\begin{aligned}
\text { P0 : } \max _{p_{k, n}, a_{k, n}} \quad & \eta_{E E}=\frac{\sum_{k=1}^{K} \sum_{n=1}^{N} a_{k, n} R_{k, n}}{P_{C}+\zeta \sum_{k=1}^{K} \sum_{n=1}^{N} a_{k, n} p_{k, n}} \\
\text { s.t. } \quad & \sum_{k=1}^{K} a_{k, n} \leq 1, \quad a_{k, n} \in\{0,1\}, \forall n \\
& \sum_{n=1}^{N} a_{k, n} p_{k, n} \leq \Psi_{k}, \quad \forall k \\
& \sum_{k=1}^{K} \lambda_{n} \beta_{n} a_{k, n} p_{k, n}\left|\widehat{h}_{n}^{S P}\right|^{2} \leq \Phi_{n}, \quad \forall n
\end{aligned}
$$

where $a_{k, n}$ is the subcarrier allocation index from SU-Tx to the kth SU-Rx in the nth subcarrier. $P_{C}$ and $\zeta$ are, respectively, the static circuit power consumption and the amplifier coefficient of SU-Tx [21]. $\Psi_{k}$ is the maximal transmission power limit of the $k$ th SU-Rx, $\Phi_{n}$ is the interference power threshold of PU in the $n$th subcarrier, and $\lambda_{n}$ is the interference tolerance factor (ITF) for subcarrier $n$.

The first constraint imposes the exclusive subcarrier allocation of OFDMA system [22]. The second constraint limits the total power allocated to each SU-Rx. The third constraint represents the interference power threshold requirement for PU's transmission, where $\lambda_{n}$ is proposed to control the interference well since $\beta_{n}$ is a probability value. The value range of $\lambda_{n}$ is $\left[1,1 / \beta_{n}\right], \lambda_{n}=1$ is the traditional algorithm, and $\lambda_{n}=1 / \beta_{n}$ means that the interference power threshold is strictly guaranteed.

According to formula (12), the uncertainty of $\widehat{g}_{k, n}$ has much greater impact on the capacity than that of $\widehat{h}_{k, n}^{P S}$. Thus, $e_{k, n}$ is modeled as stochastic-case to improve the system $\mathrm{EE}$, which is assumed to be a uniform distribution in this paper. By contrast, $e_{k, n}^{P S}$ is modeled as worst-case for simplicity and robustness; i.e., it is substituted by $\omega_{k, n}$. Besides, $e_{n}^{S P}$ is also modeled as worst-case to guarantee the interference constraint of PU in formula (13d); i.e., it is substituted by $\varphi_{n}$. As a result, the robust counterpart of $\mathbf{P 0}$ can be obtained as follows:

$$
\begin{aligned}
\text { P1: } \max _{p_{k, n}, a_{k, n}} & \eta_{E E}=\frac{\sum_{k=1}^{K} \sum_{n=1}^{N} a_{k, n} \ddot{R}_{k, n}}{P_{C}+\zeta \sum_{k=1}^{K} \sum_{n=1}^{N} a_{k, n} p_{k, n}} \\
\text { s.t. } \quad & \sum_{k=1}^{K} a_{k, n} \leq 1, \quad a_{k, n} \in\{0,1\}, \forall n \\
& \sum_{n=1}^{N} a_{k, n} p_{k, n} \leq \Psi_{k}, \quad \forall k \\
& \sum_{k=1}^{K} \lambda_{n} \beta_{n} a_{k, n} p_{k, n}\left|h_{n}^{S P}\right|^{2}\left(1+\varphi_{n}\right)^{2} \leq \Phi_{n},
\end{aligned}
$$

where

$$
\begin{aligned}
& \ddot{R}_{k, n}=\left(1-\beta_{n}\right) E\left\{\log _{2}\left(1+\frac{p_{k, n}\left|g_{k, n}\right|^{2}\left(1+e_{k, n}\right)^{2}}{\sigma_{n}^{2} \Gamma}\right\}\right. \\
& +\beta_{n} E\left\{\log _{2}(1\right. \\
& \left.+\frac{p_{k, n}\left|g_{k, n}\right|^{2}\left(1+e_{k, n}\right)^{2}}{\left(q_{k, n}^{P S}\left|h_{k, n}^{P S}\right|^{2}\left(1+\omega_{k, n}\right)^{2}+\sigma_{n}^{2}\right) \Gamma}\right\}
\end{aligned}
$$

\section{Transforming and Solving of the Problem}

In this section, since the problem $\mathbf{P 1}$ is a nonconvex optimization problem, it is converted to a convex optimization problem by a series of equivalent transformations. Then, it is solved by dual decomposition method.

4.1. Transforming of the Problem. In problem P1, the EE objective function is a nonconvex function of the power. However, it exhibits a fractional form. Thus, $\mathbf{P} \mathbf{1}$ is a factional programming problem, and it can be converted to an equivalent parameter problem given as

$$
\begin{gathered}
\text { P2: } \max _{p_{k, n}, a_{k, n}} f(\eta)=\sum_{k, n} a_{k, n} \bar{R}_{k, n} \\
-\eta\left(P_{C}+\zeta \sum_{k, n} a_{k, n} p_{k, n}\right)
\end{gathered}
$$

s.t. $(13 \mathrm{~b}),(13 \mathrm{c})$, and $(13 \mathrm{~d})$ are satisfied

where $\eta$ is a nonnegative parameter. The parameter can be interpreted as the price factor of the power consumption; i.e., the consumption of more power requires more price. According to Dinkelbach's method [23], the following result can be obtained.

Theorem 1. If and only if there is an optimal parameter $\eta^{*}$ in problem $\mathbf{P} 2$ such that $f\left(\eta^{*}\right)=0$ holds, the maximal energy efficiency of $\mathbf{P 1}$ can be achieved, which is just $\eta^{*}$.

As can be seen, problem $\mathbf{P 2}$ is a mixed-integer nonlinear optimization problem, which is NP-hard in general. Consequently, it is impractical to determine the optimal solution by exhaustive search method. To solve the optimization problem, an approach similar to the time-sharing technique can be adopted (see, e.g., [24]). The value of variable $a_{k, n}$ is relaxed to be a real number within the interval $[0,1]$, which can be regarded as the sharing proportion of time during which subcarrier $n$ is assigned to the $k$ th SU-Rx. To deal with the nonlinearity of constraints (13c) and (13d), set $x_{k, n}=a_{k, n} p_{k, n}$, which means the "actual" power allocated to subcarrier $n$ for the $k$ th SU-Rx. 
In addition, set $\Omega_{n}=\widehat{\Phi}_{n} /\left(\lambda_{n} \beta_{n}\left(1+\varphi_{n}\right)^{2}\right)$, where $\widehat{\Phi}_{n}=$ $\Phi_{n} /\left|h_{n}^{S P}\right|^{2}$ represents the relative interference power threshold (RIPT) at SU-Rx. Set $\gamma_{k, n}=\left|g_{k, n}\right|^{2} /\left(\sigma_{n}^{2} \Gamma\right)$ and $\varepsilon_{k, n}=1 /\left(\rho_{k, n}^{2}(1+\right.$ $\left.\left.\omega_{k, n}\right)^{2}+1\right)$, where $\rho_{k, n}^{2}=q_{k, n}^{P S}\left|h_{k, n}^{P S}\right|^{2} / \sigma_{n}^{2}$ represents the estimated power interference-to-noise ratio (EINR).

Then, problem $\mathbf{P} 2$ can be converted as follows:

$$
\text { P3: } \max _{p_{k, n}, a_{k, n}} f(\eta)=\sum_{k, n} a_{k, n} \bar{R}_{k, n}-\eta\left(P_{C}+\zeta \sum_{k, n} x_{k, n}\right)
$$

$$
\begin{array}{ll}
\text { s.t. } & \sum_{k=1}^{K} a_{k, n} \leq 1, \quad a_{k, n} \in[0,1], \quad \forall n \\
& \sum_{n=1}^{N} x_{k, n} \leq \Psi_{k}, \quad \forall k \\
& \sum_{k=1}^{K} x_{k, n} \leq \Omega_{n}, \quad \forall n
\end{array}
$$

where

$$
\bar{R}_{k, n}= \begin{cases}\left(1-\beta_{n}\right) E\left\{\log _{2}\left(1+\frac{x_{k, n}}{a_{k, n}} \gamma_{k, n}\left(1+e_{k, n}\right)^{2}\right)\right\}+\beta_{n} E\left\{\log _{2}\left(1+\frac{x_{k, n}}{a_{k, n}} \gamma_{k, n} \varepsilon_{k, n}\left(1+e_{k, n}\right)^{2}\right)\right\}, & a_{k, n} \neq 0 \\ 0, & \text { else }\end{cases}
$$

4.2. Solving of the Transformed Problem. Since the objective function is convex and the constraints are all linear inequalities, problem P3 is a convex optimization. Hence, it can be solved by dual decomposition method, and the Lagrangian can be given as

$$
\begin{aligned}
L= & \sum_{k, n} a_{k, n} \bar{R}_{k, n}-\eta\left(P_{C}+\zeta \sum_{k, n} x_{k, n}\right) \\
& -\sum_{n} \tau_{n}\left(\sum_{k} a_{k, n}-1\right)-\sum_{k} \delta_{k}\left(\sum_{n} x_{k, n}-\Psi_{k}\right) \\
& -\sum_{n} \mu_{n}\left(\sum_{k} x_{k, n}-\Omega_{n}\right)
\end{aligned}
$$

where $\tau_{n}, \delta_{k}$, and $\mu_{n}$ are Lagrange multipliers.

According to the dual decomposition method [25], the optimal $x_{k, n}$ and $a_{k, n}$ to maximize $L$ are the solution of problem P3. Then, the Lagrangian can be solved independently for each subcarrier.

By calculating the derivative of $L$ with regard to $x_{k, n}$, the following expression can be given:

$$
\frac{\partial L}{\partial x_{k, n}}= \begin{cases}a_{k, n} \frac{\partial \bar{R}_{k, n}}{\partial x_{k, n}}-\eta \zeta-\delta_{k}-\mu_{n}, & a_{k, n} \neq 0 \\ -\eta \zeta-\delta_{k}-\mu_{n}, & \text { else }\end{cases}
$$

where

$$
\begin{aligned}
\frac{\partial \bar{R}_{k, n}}{\partial x_{k, n}} & =\frac{1-\beta_{n}}{a_{k, n} \ln 2} A\left(\frac{x_{k, n}}{a_{k, n}}\right)+\frac{\beta_{n}}{a_{k, n} \ln 2} B\left(\frac{x_{k, n}}{a_{k, n}}\right) \\
A\left(\frac{x_{k, n}}{a_{k, n}}\right) & =E\left\{\frac{\gamma_{k, n}\left(1+e_{k, n}\right)^{2}}{1+\gamma_{k, n}\left(1+e_{k, n}\right)^{2} x_{k, n} / a_{k, n}}\right\} \\
B\left(\frac{x_{k, n}}{a_{k, n}}\right) & =E\left\{\frac{\gamma_{k, n} \varepsilon_{k, n}\left(1+e_{k, n}\right)^{2}}{1+\gamma_{k, n} \varepsilon_{k, n}\left(1+e_{k, n}\right)^{2} x_{k, n} / a_{k, n}}\right\}
\end{aligned}
$$

If $a_{k, n}=0$, then $L$ is a decreasing function of $x_{k, n}$; hence the optimal value of $x_{k, n}$ is zero which maximizes $L$. If $a_{k, n} \neq$ 0 , then $\partial L / \partial x_{k, b}$ is a decreasing function of $x_{k, b}$ according to formulas (20) and (21). Thus, $L$ can achieve the maximum value when $\partial L / \partial x_{k, b}$ is zero; i.e.,

$$
a_{k, n} \frac{\partial \bar{R}_{k, n}}{\partial x_{k, n}}=\eta \zeta+\delta_{k}+\mu_{n}
$$

Substituting formula (21) into (24), the following equation is satisfied:

$$
\ln 2\left(\eta \zeta+\delta_{k}+\mu_{n}\right)=Q\left(p_{k, n}^{*}\right)
$$

where $Q\left(p_{k, n}^{*}\right)=\left(1-\beta_{n}\right) A\left(p_{k, n}^{*}\right)+\beta_{n} B\left(p_{k, n}^{*}\right) \cdot p_{k, n}^{*}=x_{k, n}^{*} / a_{k, n}$ and $x_{k, n}^{*}$ are the optimal values of $p_{k, n}$ and $x_{k, n}$, respectively.

Assuming that $e_{k, n}$ is a uniform distribution bounded by formula (9), the following equation is satisfied:

$$
\begin{aligned}
& A\left(p_{k, n}^{*}\right)=\int_{-\theta_{k, n}}^{\theta_{k, n}} \frac{\gamma_{k, n}\left(1+e_{k, n}\right)^{2}}{2 \theta_{k, n}\left(1+p_{k, n}^{*} \gamma_{k, n}\left(1+e_{k, n}\right)^{2}\right)} d e_{k, n} \\
& \text { If } p_{k, n}^{*}=0 \text {, then } \\
& A(0)=\frac{\gamma_{k, n}}{6 \theta_{k, n}}\left(\left(1+\theta_{k, n}\right)^{3}-\left(1-\theta_{k, n}\right)^{3}\right)
\end{aligned}
$$

Otherwise

$$
A\left(p_{k, n}^{*}\right)=\frac{1}{p_{k, n}^{*}}-\frac{C-D}{2 \theta_{k, n} p_{k, n}^{*} \sqrt{p_{k, n}^{*} \gamma_{k, n}}}
$$

where $C=\arctan \left(\sqrt{p_{k, n}^{*} \gamma_{k, n}}\left(1+\theta_{k, n}\right)\right)$ and $D=$ $\arctan \left(\sqrt{p_{k, n}^{*} \gamma_{k, n}}\left(1-\theta_{k, n}\right)\right)$. 
Similarly, if $p_{k, n}^{*}=0$, then

$$
B(0)=\frac{\gamma_{k, n} \varepsilon_{k, n}}{6 \theta_{k, n}}\left(\left(1+\theta_{k, n}\right)^{3}-\left(1-\theta_{k, n}\right)^{3}\right)
$$

Otherwise

$$
B\left(p_{k, n}^{*}\right)=\frac{1}{p_{k, n}^{*}}-\frac{E-F}{2 \theta_{k, n} p_{k, n}^{*} \sqrt{p_{k, n}^{*} \gamma_{k, n} \varepsilon_{k, n}}}
$$

where $E=\arctan \left(\sqrt{p_{k, n}^{*} \gamma_{k, n} \varepsilon_{k, n}}\left(1+\theta_{k, n}\right)\right)$ and $F=$ $\arctan \left(\sqrt{p_{k, n}^{*} \gamma_{k, n} \varepsilon_{k, n}}\left(1-\theta_{k, n}\right)\right)$.

Since the power value cannot be negative, the optimal value can be given as

$$
p_{k, n}^{*}= \begin{cases}0, & Q(0) \leq \ln 2\left(\eta \zeta+\delta_{k}+\mu_{n}\right) \\ p_{k, n}^{*}: Q\left(p_{k, n}^{*}\right)=\ln 2\left(\eta \zeta+\delta_{k}+\mu_{n}\right), & \text { else }\end{cases}
$$

According to formulas (26)-(30), $Q\left(p_{k, n}^{*}\right)$ is a nonlinear and decreasing function of $p_{k, n}^{*}$. Thus, equation (31) has only one solution and can be solved by Newton-Raphson method with a certain amount of computational effort [26].

Then, the Lagrangian of (19) can be modified as

$$
\begin{aligned}
L= & -\eta P_{C}+\sum_{k} \delta_{k} \Psi_{k}+\sum_{n} \mu_{n} \Omega_{n}+\sum_{n} \tau_{n}\left(1-\sum_{k} a_{k, n}\right) \\
& +\sum_{k, n} a_{k, n} Y_{k, n}
\end{aligned}
$$

where

$$
\begin{aligned}
Y_{k, n}= & \bar{R}_{k, n}^{*}-\left(\eta P_{C}+\delta_{k}+\mu_{n}\right) p_{k, n}^{*} \\
\bar{R}_{k, n}^{*}= & \left(1-\beta_{n}\right) E\left\{\log _{2}\left(1+p_{k, n}^{*} \gamma_{k, n}\left(1+e_{k, n}\right)^{2}\right)\right\} \\
& +\beta_{n} E\left\{\log _{2}\left(1+p_{k, n}^{*} \gamma_{k, n} \varepsilon_{k, n}\left(1+e_{k, n}\right)^{2}\right)\right\}
\end{aligned}
$$

As seen in (32), $\sum_{k, n} a_{k, n} Y_{k, n}$ must be maximized in order to maximize $L$, while the Lagrange multiplier $\tau_{n}$ is used to satisfy $\sum_{k} a_{k, n} \leq 1$. So, for each $n$, if the value of $Y_{k, n}$ is the maximum in all users, then let $a_{k, n}=1$; otherwise $a_{k, n}=0$. It means that subcarrier $n$ is completely allocated to the best user $k$; i.e.,

$$
k_{n}^{*}=\arg \max _{k}\left(Y_{k, n}\right)
$$

As a result, the optimal power assignment to maximize $L$ can be formulated as follows:

$$
\begin{aligned}
& a_{k, n}^{*}= \begin{cases}1 & k=k_{n}^{*} \\
0 & \text { else }\end{cases} \\
& p_{k, n}^{*}= \begin{cases}p_{k, n}^{*} & a_{k, n}^{*}=1 \\
0 & \text { else }\end{cases}
\end{aligned}
$$

Theorem 2. Equation (37) is the optimal solution to problem P2.
Proof. Since problem P3 is a convex problem, the duality gap of the Lagrangian in (19) is zero, which indicates that equation (37) is the optimal solution to P3. Meanwhile, P3 is the relaxation of $\mathbf{P 2}$; thus (37) is the upper bound on the solution to P2. Moreover, (37) satisfies all the constraints of P2. Therefore, it is also the optimal solution to $\mathbf{P 2}$.

\section{Algorithm Implementation}

Equation (37) is the optimal solution for given Lagrange multipliers and parameter $\eta$. To realize the EE maximization algorithm, the key is to find the optimal values of the Lagrange multipliers and parameter $\eta$. The multipliers can be updated by the following subgradient methods:

$$
\begin{aligned}
& \delta_{k}^{t+1}=\left[\delta_{k}^{t}-d_{1}\left(\Psi_{k}-\sum_{n} p_{k, n}^{*}\right)\right]^{+} \\
& \mu_{n}^{t+1}=\left[\mu_{n}^{t}-d_{2}\left(\Omega_{n}-\sum_{k} p_{k, n}^{*}\right)\right]^{+}
\end{aligned}
$$

where $d_{1}=z_{1} / \sqrt{t+1}$ and $d_{2}=z_{2} / \sqrt{t+1}$ are the iteration step size of $\delta_{k}$ and $\mu_{n}$, respectively; $[\cdot]^{+}$denotes $\max (\cdot, 0)$; $z_{1}$ and $z_{2}$ are the step size control coefficients; and $t$ is the iteration index.

As seen in Theorem 1 , the parameter $\eta$ can be solved by the well-known Dinkelbach's method, and it can be converged to the optimal value with a superlinear convergence rate.

The proposed EE maximization power allocation algorithm is shown in Algorithm 1.

The complexity of the proposed algorithm is as follows. Let $I_{p}$ denote the number of zero-finding iterations for equation (31) by Newton-Raphson method, and let $I_{c}$ denote the number of evaluations for formula (34); then the number of operations in line 4 is $o\left(K N\left(I_{p}+I_{c}\right)\right)$. Let $I_{\delta}$ and $I_{\mu}$ denote the number of iterations in lines 7 and 8 to be converged, respectively. Then, the number of operations from line 4 to 10 is $o\left(K N\left(I_{p}+I_{c}\right) \max \left(I_{\delta}, I_{\mu}\right)\right)$. Since the most complex variable $\bar{R}_{k, n}^{*}$ has already been obtained which is hidden in line 4 , the number of operations in line 13 can be neglected. Let $I_{\eta}$ denote the iteration number of $\eta$; then the overall 


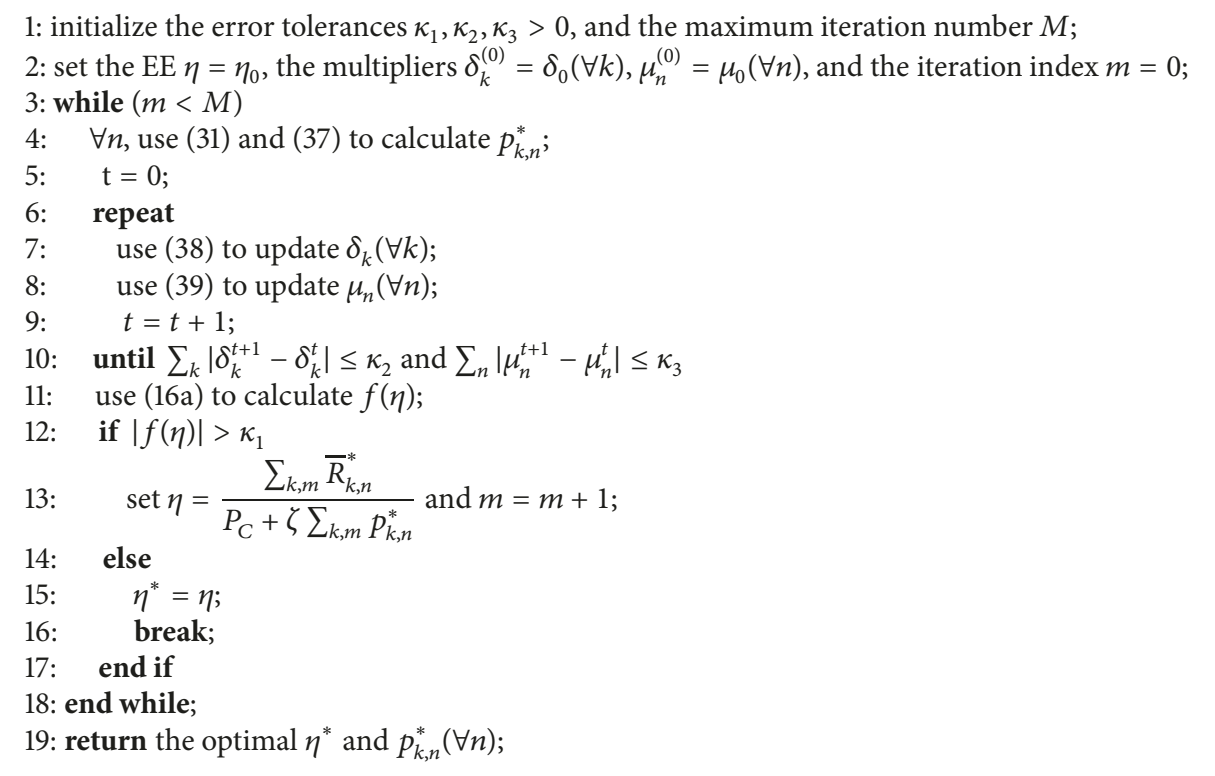

Algorithm 1: Energy efficient maximization power allocation algorithm.

complexity is $o\left(K N I_{\eta}\left(I_{p}+I_{c}\right) \max \left(I_{\delta}, I_{\mu}\right)\right)$. The proposed algorithm is linear in terms of the number of subcarriers, while the traditional exhaustive search is exponential.

\section{Simulation and Analysis}

In this section, simulation results are presented to evaluate the performance of the proposed EE maximization algorithm. The simulation parameters of the UAN environment are consistent with [17]. The acoustic band $B$ is $20-30 \mathrm{KHz}$ with a number of $J=64$ subcarriers. The maximum Doppler shift $\Delta f_{l}$ is set to be $1 \mathrm{~Hz}$, and the delay $\tau_{l}$ lies in the range $0-0.01 \mathrm{~s}$. The spreading factor $s$ is set to be 1.5 for the so-called practical spreading. The noise constant level $\chi_{0}$ is taken to be $50 \mathrm{~dB}$ for the quiet deep sea. The target BER is set to be $1 \times 10^{-3}$, which can basically meet the data transmission requirement [27]. For the cognitive system, the number of the SU receivers (i.e., $K$ ) is set to be 4 . The probability that the subcarrier is actually occupied by PU (i.e., $P\left(O_{n}\right)$ ) is set to be 0.5 ; then the idle subcarriers can be obtained by calculating $P\left(\widehat{I}_{n}\right)$ in formula (5), and the number $N$ can be available. The static circuit power consumption $P_{C}$ and amplifier coefficient $\zeta$ are set to be $0.02 \mathrm{~W}$ and 0.2 , respectively. The EINR is set to be 10 $\mathrm{dB}$ by considering the power interference coming from PUTx. Monte Carlo simulation times are $10^{4}$.

Figure 2 illustrates the system EE versus the RIPT under different power limits at each SU-Rx. The transmission distance is set as $1 \mathrm{~km}$, the USIC 0.1 , the ITF 1 , the detection probability 0.9 , and the false-alarm probability 0.1 . As can be seen, the EE curves under different power limits are close when the RIPT is low, because the SU-Tx can only transmit power with low level. With the increase of the RIPT, the EE under higher power limit is larger than that under lower power limit. It can be explained that the power allocation

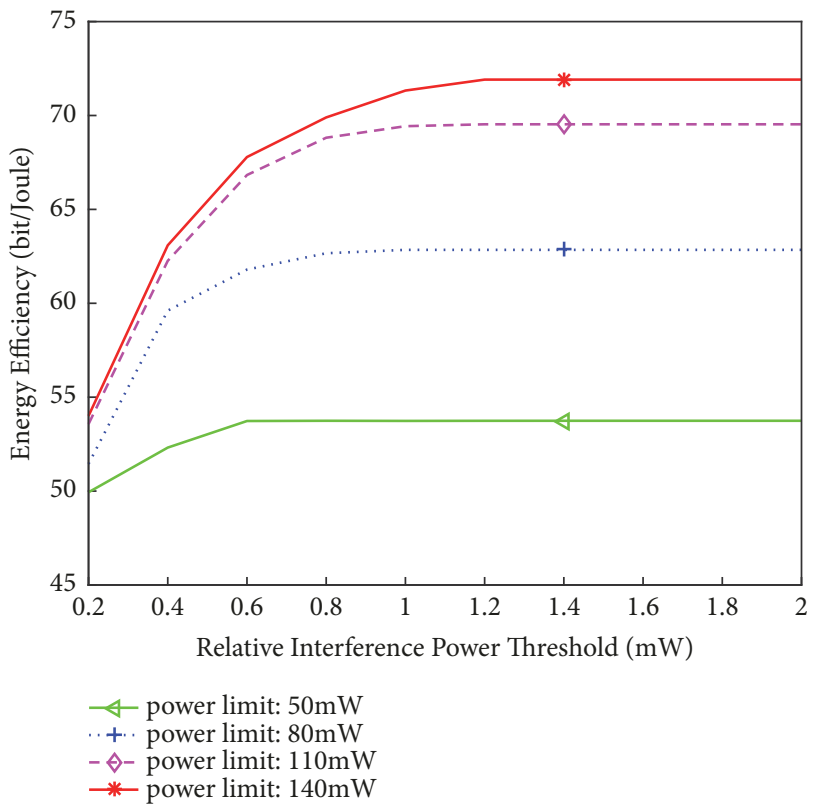

FIGURE 2: The system EE versus the RIPT under different power limits.

strategy is more flexible under higher power limit. When the RIPT becomes sufficiently large, the EE remains the same, because the optimal power is lower than the RIPT.

Figure 3 shows the system EE versus the ITF under different detection probability and false-alarm probability, where the RIPT is set as $1 \mathrm{~mW}$ and the power limit is set as $80 \mathrm{~mW}$. According to formula (5), when the detection probability and false-alarm probability are, respectively, set as $P_{d}=0.9, P_{f a}=0.1 ; P_{d}=0.9, P_{f a}=0.3$ and $P_{d}=0.825, P_{f a}=$ 


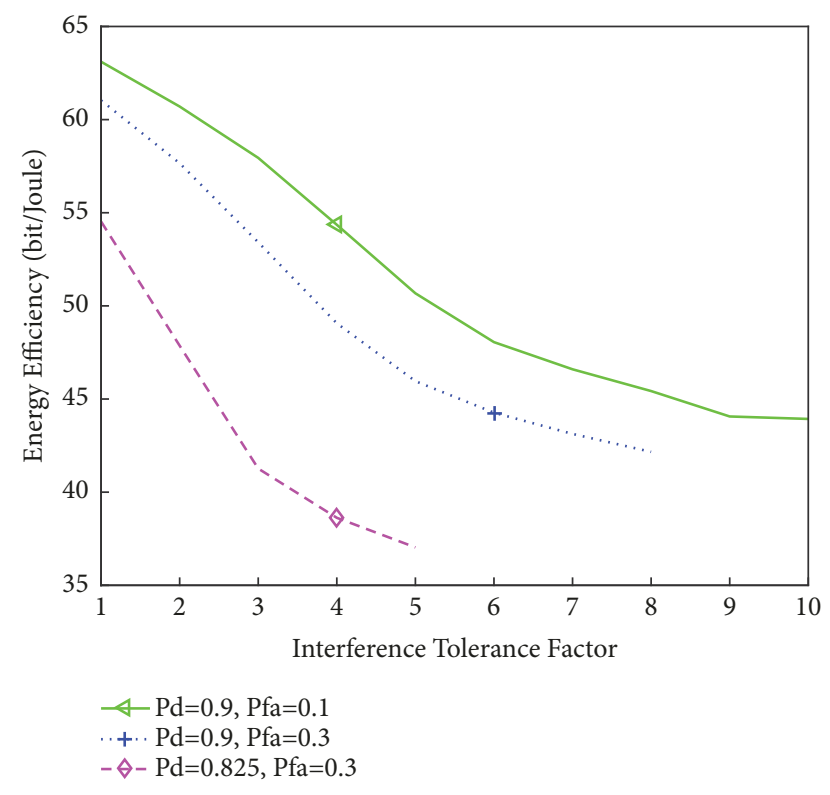

FIgURE 3: The system EE versus the ITF under different detection probability and false-alarm probability.

0.3 , the probability of the spectrum sensing errors (i.e., $\forall \beta_{n}$ ) is $0.1,0.125$, and 0.2 , correspondingly. As can be seen, the $\mathrm{EE}$ is smaller with the increase of the spectrum sensing errors, because the interference coming from $\mathrm{PU}$ is more serious. Traditional methods (i.e., the ITF is set as 1) can only meet the RIPT in statistics but cannot absolutely guarantee it. To completely solve the problem, the ITF needs to be set as 10,8 , and 5, respectively. However, it can be seen that the EE gets smaller with the increase of the ITF, because the interference power constraint gets stricter.

In Figure 4, the quality-of-service (QoS) satisfying probability of PU (QSPP) versus the ITF is evaluated under the same conditions as Figure 3, where QSPP means the probability that the interference from $\mathrm{SU}$ to $\mathrm{PU}$ is less than the interference power threshold of PU. As can be seen, the QSPP under lower $\beta_{n}$ is smaller than that under higher $\beta_{n}$. It can be explained that the statistical probability value of the RIPT is smaller for lower $\beta_{n}$; thus the RIPT constraint cannot be guaranteed well when the subcarrier is actually occupied by PU. The QSPP gets larger with the increase of the ITF, because the interference power constraint gets more strict. In a word, the ITF can be flexibly adjusted to balance the EE and the interference from SU to PU.

Figure 5 shows the system EE versus the transmission distance under different power limits, where the RIPT is set as $1 \mathrm{~mW}$, the ITF 2 , the detection probability 0.9 , and the falsealarm probability 0.1 . It can be observed that the EE under higher power limit is larger than that under lower power limit, and the performance gap diminishes as the power limit increases, which is consistent with Figure 2. Clearly, the EE suffers a degradation with the increase of the distance, because the SNR gets worse.

In Figure 6, the system capacity versus the distance is evaluated under the same conditions as Figure 5. As can be

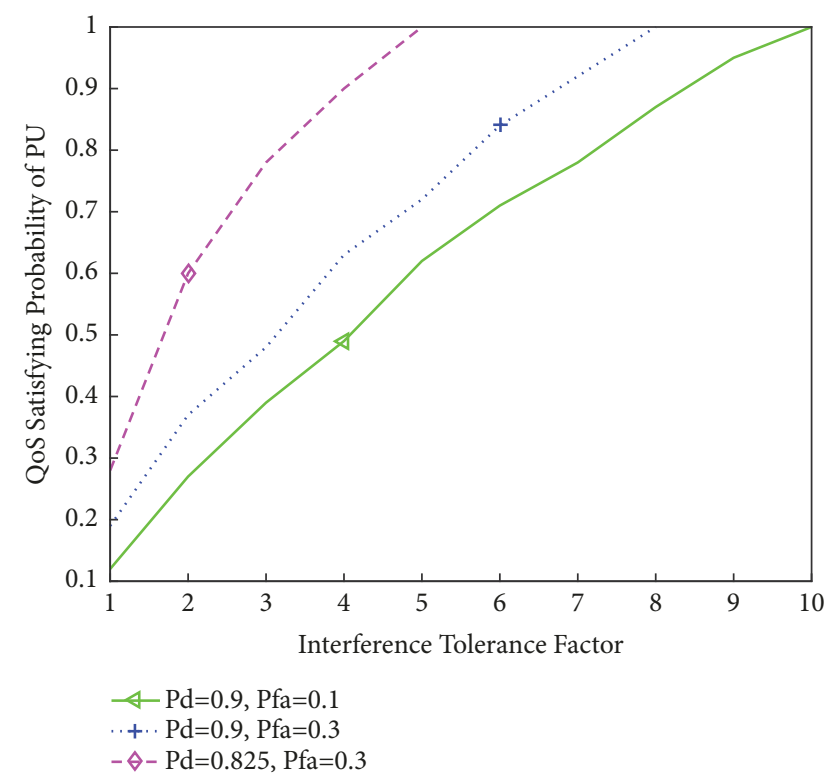

FIGURE 4: The QoS satisfying probability of PU versus the ITF under different detection probability and false-alarm probability.

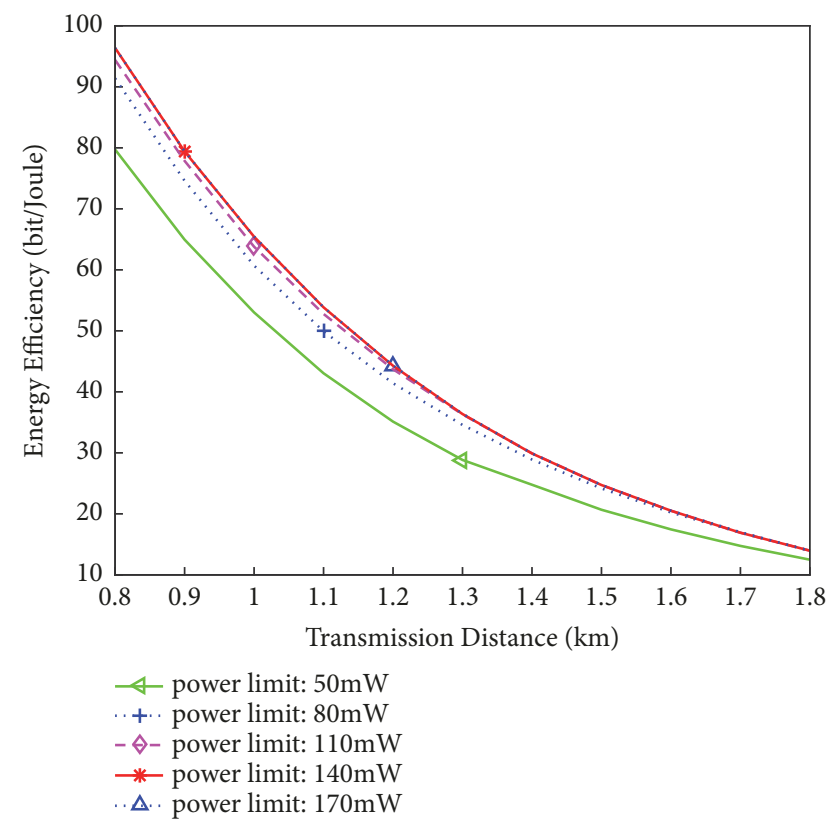

FIGURE 5: The system EE versus the transmission distance under different power limits.

seen, the traces of the curves are similar to Figure 5. The difference is that the gap of different curves is relatively larger than that of Figure 5, because more power is consumed for higher capacity at the same channel.

The system EE with different USIC is assessed in Figure 7, where the transmission distance is set as $1 \mathrm{~km}$, the RIPT $1 \mathrm{~mW}$, the ITF 2 , and the power limit $80 \mathrm{~mW}$. The notations $S S, S P$, and $P S$ in the figure mean the channel uncertainty from SU-Tx to SU-Rx, SU-Tx to PU-Rx, and PU-Tx to SU$\mathrm{Rx}$, respectively. To clearly assess the impact of each type 


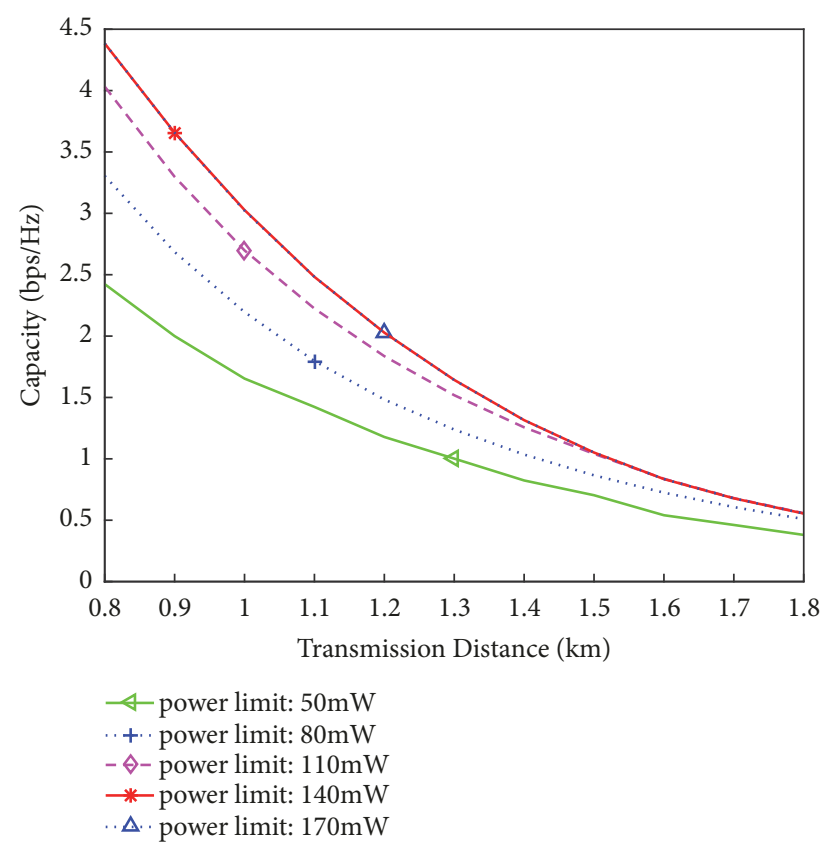

FIGURE 6: The system capacity versus the transmission distance under different power limits.

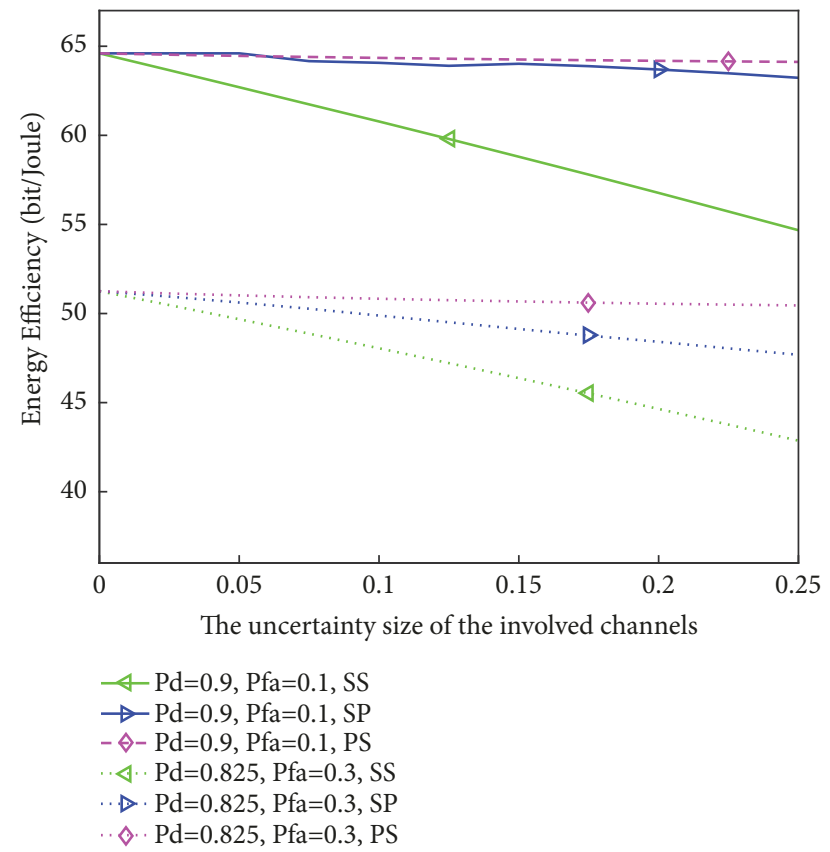

FIgure 7: The system EE with different USIC.

of channel uncertainty on the EE, each curve in the figure only considers the uncertainty of the noted channel and ignores the uncertainties of others. As can be seen, the EE gets larger when the probability of the spectrum sensing errors decreases, which is consistent with Figure 3. Moreover, with the increase of the USIC, the channel uncertainty from SU-Tx to SU-Rx has the most serious impact on the EE. It is reasonable according to formula (15); the capacity is

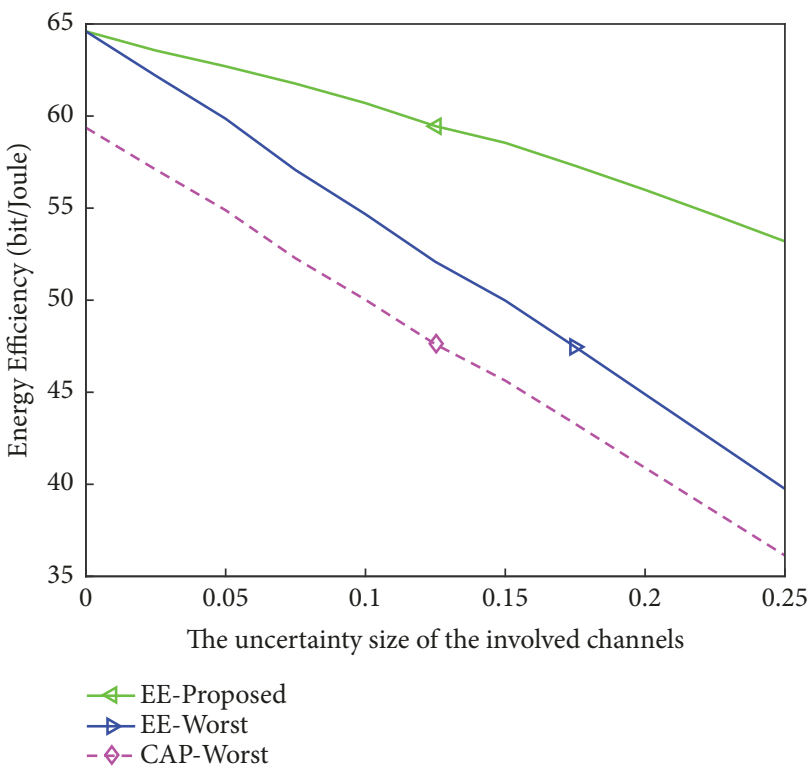

FIGURE 8: System EE comparison of three algorithms with different USIC.

determined by the channel uncertainty from SU-Tx to SU$\mathrm{Rx}$. The channel uncertainty from SU-Tx to PU-Rx has the second most serious impact on the EE, because the interference power constraint gets more strict by considering the worst-case.

Figure 8 compares the system EE of the proposed algorithm with others under the same conditions as Figure 7, except that the RIPT is set as $0.5 \mathrm{~mW}$ and the ITF 1 due to the requirement of the consistent algorithmic environment. The notations EE-Worst and CAP-Worst in the figure mean the EE maximization algorithm in [12] and capacity maximization algorithm in [15], respectively. To compare the performance of the algorithms fairly, the spectrum sensing errors are considered in the EE-Worst and are consistent with those of other algorithms. It is observed that the EE of the proposed algorithm decreases slowly with the increase of USIC, while that of the two others decreases relatively rapidly. Because the channel uncertainty from SU-Tx to SU-Rx in the proposed scheme is modeled as stochastic-case other than worst-case, the EE has been improved by solving the expectation of CSI correlation function. Furthermore, there is a performance gap between the CAP-Worst and the two others because the former optimizes the capacity rather than the EE.

\section{Conclusions}

Based on the spectrum sensing errors and CSI uncertainties, an adaptive resource allocation algorithm for CUAN is proposed to maximize the system EE. By taking the spectrum sensing errors into account, we add power interference from PU-Tx to SU-Rx in the objective function. Meanwhile, interference tolerance factor is introduced to well control the interference from SU-Tx to PU-Rx, which is evaluated with 
the QoS satisfying probability of PU. Since the channel uncertainty from SU-Tx to SU-Rx has the most serious impact on the EE, it is modeled as stochastic-case other than worst-case. By solving the expectation of CSI correlation, system EE is increased significantly. Simulation results verify the validity of the proposed algorithm. Our future research work will focus on cross-layer resource allocation techniques for $\mathrm{EE}$ maximization schemes in the imperfect CUAN environment via acknowledge/not-acknowledge (ACK/NAK) feedback.

\section{Data Availability}

The Matlab Source Code data used to support the findings of this study are available from the corresponding author upon request.

\section{Conflicts of Interest}

The authors declare that they have no conflicts of interest.

\section{Acknowledgments}

This work was supported by Zhejiang Provincial Natural Science Foundation of China under Grants LY18F010001 and LY18F010010 and the Natural Science Foundation of China under Grant 61571250.

\section{References}

[1] D. Pompili and I. F. Akyildiz, "Overview of networking protocols for underwater wireless communications," IEEE Communications Magazine, vol. 47, no. 1, pp. 97-102, 2009.

[2] H. Tan, W. K. Seah, and L. Doyle, "Exploring cognitive techniques for bandwidth management in integrated underwater acoustic systems," in Proceedings of the IEEE Oceans, pp. 126132, Kobe, Japan, September 2008.

[3] A. Goldsmith, S. A. Jafar, I. Maric, and S. Srinivasa, "Breaking spectrum gridlock with cognitive radios: an information theoretic perspective," Proceedings of the IEEE, vol. 97, no. 5, pp. 894-914, 2009.

[4] D. Darsena, G. Gelli, and F. Verde, "Convolutive superposition for multicarrier cognitive radio systems," IEEE Journal on Selected Areas in Communications, vol. 34, no. 11, pp. 2951-2967, 2016.

[5] W. Liang, S. X. Ng, and L. Hanzo, "Cooperative overlay spectrum access in cognitive radio networks," IEEE Communications Surveys \& Tutorials, vol. 19, no. 3, pp. 1924-1944, 2017.

[6] L. Jing, C. He, J. Huang, and Z. Ding, "Energy management and power allocation for underwater acoustic sensor network," IEEE Sensors Journal, vol. 17, no. 19, pp. 6451-6462, 2017.

[7] J. Mao, G. Xie, J. Gao, and Y. Liu, "Energy efficiency optimization for ofdm-based cognitive radio systems: a water-filling factor aided search method," IEEE Transactions on Wireless Communications, vol. 12, no. 5, pp. 2366-2375, 2013.

[8] C. Xu, M. Sheng, C. Yang, X. Wang, and L. Wang, "Pricingbased multiresource allocation in OFDMA cognitive radio networks: An energy efficiency perspective," IEEE Transactions on Vehicular Technology, vol. 63, no. 5, pp. 2336-2348, 2014.

[9] F. A. de Souza, B. S. Chang, R. D. Souza, G. Brante, and M. E. Pellenz, "Modulation order optimization for energy efficient underwater acoustic communications," in Proceedings of the IEEE Oceans, pp. 1-4, Monterey, USA, September 2016.

[10] X. Zhao, D. Pompili, and J. Alves, "Underwater acoustic carrier aggregation: Achievable rate and energy-efficiency evaluation," IEEE Journal of Oceanic Engineering, vol. 42, no. 4, pp. 10351048, 2017.

[11] S.-J. Kim, N. Y. Soltani, and G. B. Giannakis, "Resource allocation for OFDMA cognitive radios under channel uncertainty," IEEE Transactions on Wireless Communications, vol. 12, no. 7, pp. 3578-3587, 2013.

[12] L. Wang, M. Sheng, Y. Zhang, X. Wang, and C. Xu, "Robust energy efficiency maximization in cognitive radio networks: the worst-case optimization approach," IEEE Transactions on Communications, vol. 63, no. 1, pp. 51-65, 2015.

[13] S. M. Almalfouh and G. L. Stüber, "Interference-aware radio resource allocation in OFDMA-based cognitive radio networks," IEEE Transactions on Vehicular Technology, vol. 60, no. 4, pp. 1699-1713, 2011.

[14] H. Fathi and S. M.-S. Sadough, "Robust power and subcarrier allocation for OFDM-based cognitive radio networks considering spectrum sensing uncertainties," Radioengineering, vol. 22, no. 3, pp. 810-817, 2013.

[15] L. Zhu and X. Zhao, "Robust power allocation for orthogonal frequency division multiplexing-based overlay/underlay cognitive radio network under spectrum sensing errors and channel uncertainties," IET Communications, vol. 10, no. 15, pp. 20102017, 2016.

[16] M. Biagi, A. Petroni, S. Colonnese, R. Cusani, and G. Scarano, "On rethinking cognitive access for underwater acoustic communications," IEEE Journal of Oceanic Engineering, vol. 41, no. 4, pp. 1045-1060, 2016.

[17] C. Polprasert, J. A. Ritcey, and M. Stojanovic, "Capacity of OFDM systems over fading underwater acoustic channels," IEEE Journal of Oceanic Engineering, vol. 36, no. 4, pp. 514-524, 2011.

[18] G. Zhou, Y. Li, Y. He, X. Wang, and M. Yu, "Artificial fish swarm based power allocation algorithm for MIMO-OFDM relay underwater acoustic communication," IET Communications, vol. 12, no. 9, pp. 1079-1085, 2018.

[19] J. M. Jornet, M. Stojanovic, and M. Zorzi, "On joint frequency and power allocation in a cross-layer protocol for underwater acoustic networks," IEEE Journal of Oceanic Engineering, vol. 35, no. 4, pp. 936-947, 2010.

[20] Y. Xu, X. Zhao, and Y.-C. Liang, "Robust power control and beamforming in cognitive radio networks: a survey," IEEE Communications Surveys \& Tutorials, vol. 17, no. 4, pp. 18341857, 2015.

[21] D. W. K. Ng, E. S. Lo, and R. Schober, "Energy-efficient resource allocation in multi-cell OFDMA systems with limited backhaul capacity," IEEE Transactions on Wireless Communications, vol. 11, no. 10, pp. 3618-3631, 2012.

[22] B. Li, J. Huang, S. Zhou et al., "MIMO-OFDM for high-rate underwater acoustic communications," IEEE Journal of Oceanic Engineering, vol. 34, no. 4, pp. 634-644, 2009.

[23] F. Zhou, N. C. Beaulieu, Z. Li, J. Si, and P. Qi, "Energy-efficient optimal power allocation for fading cognitive radio channels: ergodic capacity, outage capacity, and minimum-rate capacity," IEEE Transactions on Wireless Communications, vol. 15, no. 4, pp. 2741-2755, 2016. 
[24] H. Al-Tous and I. Barhumi, "Resource allocation for multiplesources single-relay cooperative communication OFDMA systems," IEEE Transactions on Mobile Computing, vol. 15, no. 4, pp. 964-981, 2016.

[25] D. P. Palomar and M. Chiang, "A tutorial on decomposition methods for network utility maximization," IEEE Journal on Selected Areas in Communications, vol. 24, no. 8, pp. 1439-1451, 2006.

[26] Z. Shen, J. G. Andrews, and B. L. Evans, "Adaptive resource allocation in multiuser OFDM systems with proportional rate constraints," IEEE Transactions on Wireless Communications, vol. 4, no. 6, pp. 2726-2737, 2005.

[27] A. Doosti-Aref and A. Ebrahimzadeh, "Adaptive relay selection and power allocation for ofdm cooperative underwater acoustic systems," IEEE Transactions on Mobile Computing, vol. 17, no. 1, pp. 1-15, 2018. 


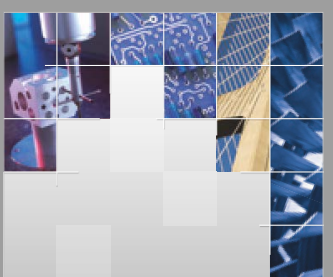

\section{Enfincering}
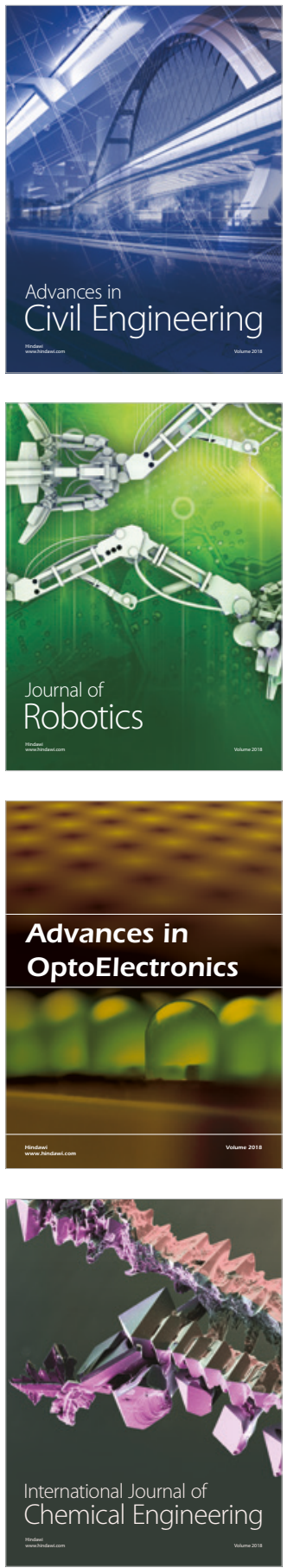

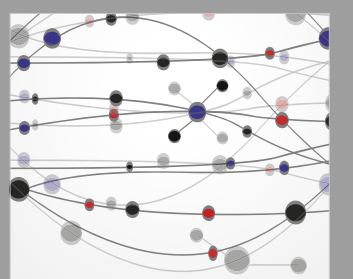

\section{Rotating \\ Machinery}

The Scientific World Journal

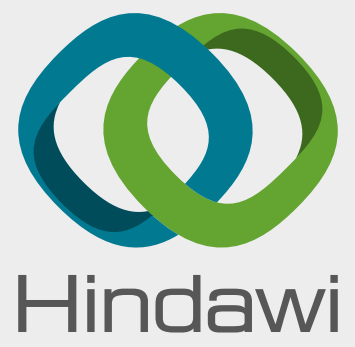

Submit your manuscripts at

www.hindawi.com
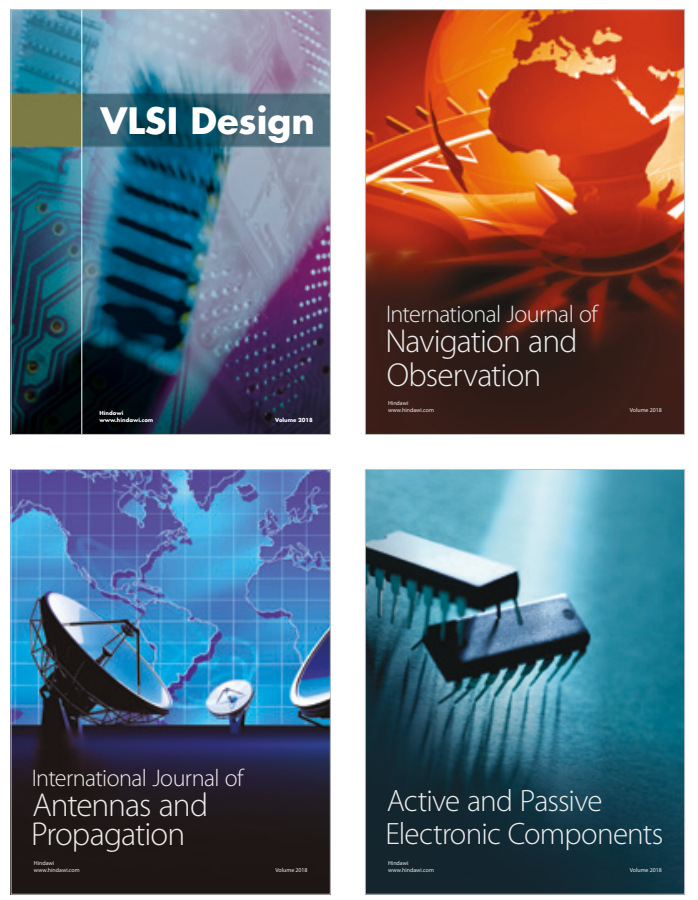
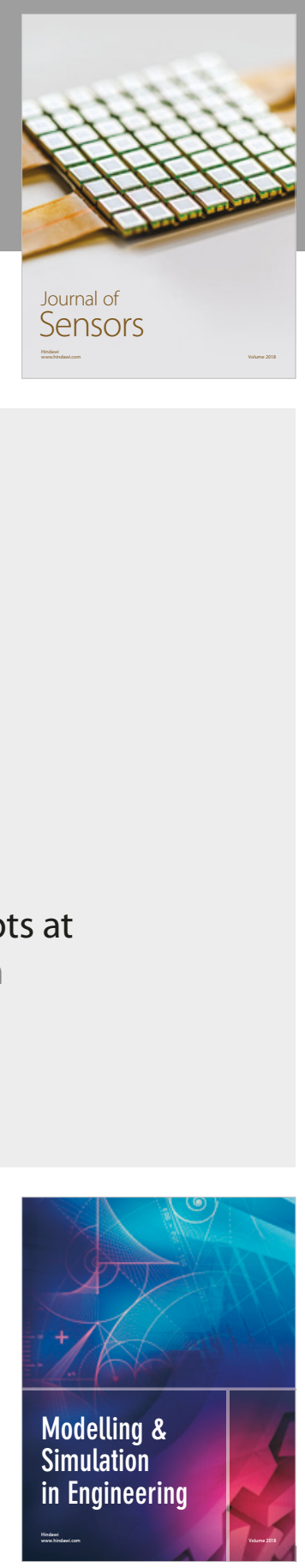

\section{Advances \\ Multimedia}
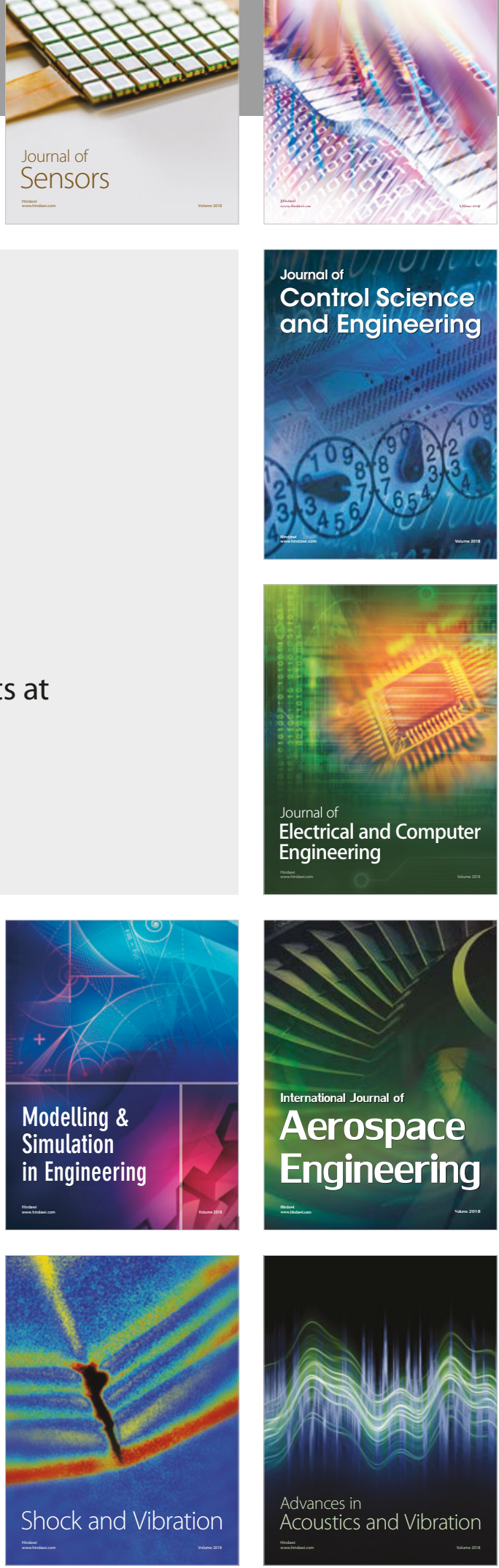